\title{
Five-axis Spline Interpolation Algorithm for Digital Manufacturing System
}

\author{
LI Huiying ${ }^{1, ~ a}$ and CHEN Liangji ${ }^{2, b}$ * \\ ${ }^{1}$ Zhengzhou Institute of Aeronautical Industry Management, China \\ ${ }^{2}$ Zhengzhou Institute of Aeronautical Industry Management, China

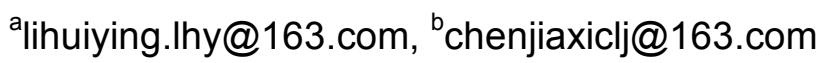 \\ * corresponding author
}

Keywords: NURBS curve, location and federate controlling, cutter location data

Abstract. At present, Non-Uniform Rational B-Spline(NURBS) curves are mostly used to generate arbitrary contour of a complex shape in the CAD field. In this article, a new method with NURBS technology is presented and realized in a location and federate controlling system. In the method, the tool paths and the cutter locations(CL) were represented into NURBS curves based on the same knot vector. The above CL data was firstly calculated and then transformed to five motion commands of five axes of machine through the real-time post-procession algorithm. The acceleration/deceleration controlling method is also presented to avoid the impact of machine. The proposed 5-axis spline interpolation method is realized and the experimental result of machining shows that the method is valid.

\section{Introduction}

Till now, most of complex products such as aeronautical turbine blades, impellors, dies, molds etc. are machined on 5-axis Computer Numerical Controlled (CNC) machines. The core of the machines is the contour controlling system which decides machining efficiency and accuracy of a part. However, the current 5-axis CNC system mostly provide only line interpolators, that is, tool motion along straight lines is supported. To machine a surface, the tool paths, which are also known as the cutter location (CL) paths, are typically approximated with piecewise linear segments by CAD/CAM systems. The approximately machining method has its own drawbacks in advancing efficiency and accuracy of a machined part. To overcome the disadvantages, it must be taken into account that the 5-axis control system has intelligent ability to generate the tool paths of the machined surfaces.

Non-Uniform Rational B-Spline (NURBS), as the only standard of data-exchange of product sanctioned by ISO, has been mostly used in almost all CAD/CAM systems to describe work-piece's surface. Based on the reality, the CNC system should be provided with the ability to directly generate 5 -axis NURBS curves or surfaces in real-time.

Some NURBS interpolation methods had been proposed by several investigators[2-6]. Cheng et al.[4] had proposed a real-time NURBS curve motion command generator for CNC machines. Zhiming et al.[6] developed a NURBS curve interpolator for CNC machining based on the geometric properties of the tool path. However, most of them had concentrated their attentions on 3-axis ball-end machining, but for 5-axis NURBS surface interpolator, little has been done.

When developing and realizing a 5-axis CNC system with function of NURBS interpolation, related study must be carried out. In this paper, a real-time 5-axis NURBS curve interpolation method is given and also its' format of G code.

\section{NURBS Method}

A $p$-degree NURBS curve with parameter $u$ can be defined as follows: 


$$
\boldsymbol{C}(u)=[x(u) y(u) z(u)]^{\mathrm{T}}=\frac{\sum_{i=0}^{n} N_{i, p}(u) W_{i} \boldsymbol{P}_{i}}{\sum_{i=0}^{n} N_{i, p}(u) W_{i}}
$$

where $\boldsymbol{P}_{i}$ is the $i^{\text {th }} 3$-D control point; $W_{i}$ is the corresponding weight factor of $\boldsymbol{P}_{i} ;(n+1)$ is the number of control points; $N_{i, p}(u)$, B-spline basis function with degree of $p$, can be calculated by the following formula:

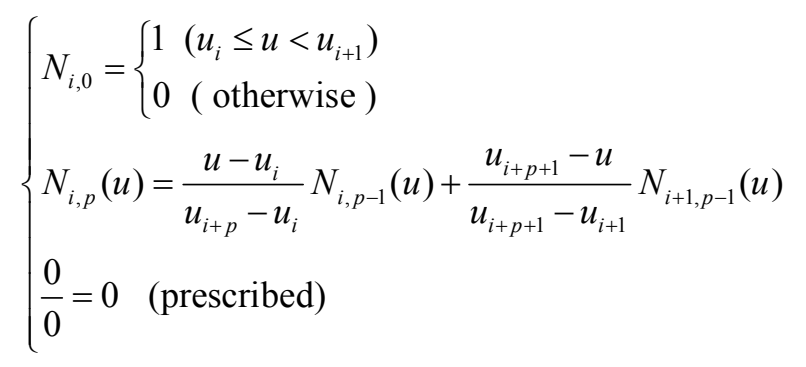

where $\left[u_{i}, \ldots, u_{i+p+1}\right]$ is the knot vector. More information about NURBS can be found in Ref.[1].

\section{Location Controlling Method}

The task of real-time NURBS spline interpolation is to calculate next interpolation period's CL data that including tool-tip location coordination and tool-axis orientation and then transfer the CL data into machine tool's motion commands such as $X, Y, Z, A, C$ through post-procession.

As shown in Fig. $1, \boldsymbol{C}_{0}(u)$ and $\boldsymbol{C}_{1}(u)$ is the cutter center point spline and the orientation spline, respectively. The two splines are constructed in the same knot vector. Assumed that parameter $u$ is function of time $t$, that is, $u=u(t)$. By using Taylor's expansion of the parameter $u$ with respect to time $t$ to obtain the first order approximation interpolation algorithm, the first order approximation up to the first derivatives is

$$
u_{k+1}=u_{k}+\left.T_{\mathrm{s}} \frac{\mathrm{d} u}{\mathrm{~d} t}\right|_{t=t_{k}}
$$

where $T_{\mathrm{s}}$ is interpolation period, $u_{k}$ and $u_{k+1}$ are corresponding parameters of current and next time $t_{k}$ and $t_{k+1}$.

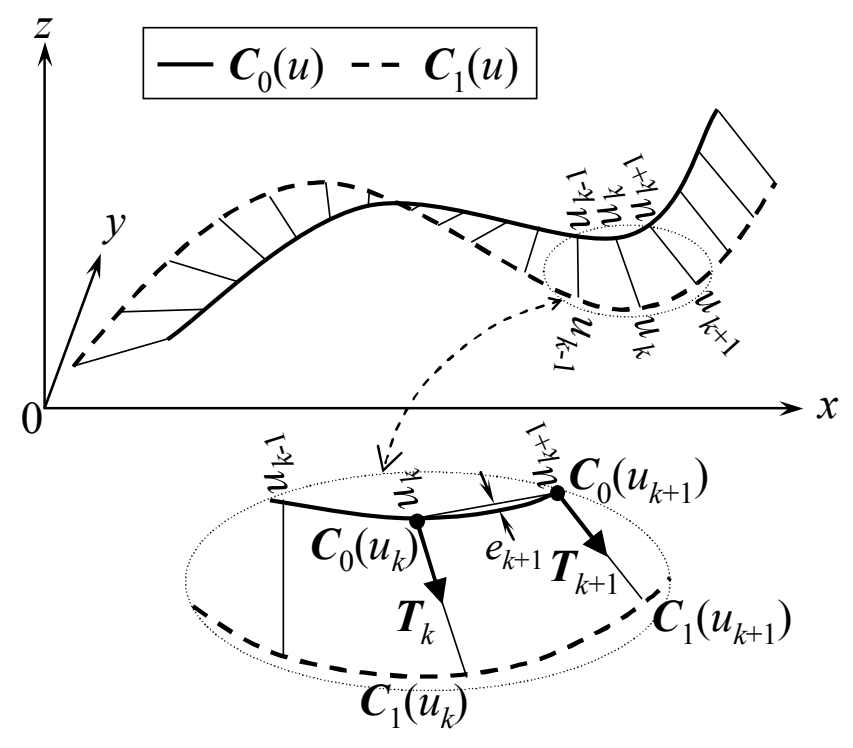

Figure 1. Principle of NURBS interpolation method

The federate of tool-tip point along the curve $\boldsymbol{C}_{0}(u)$ is defined by 


$$
\boldsymbol{V}(u)=\frac{\mathrm{d} \boldsymbol{C}_{0}(u)}{\mathrm{d} t}=\left(\frac{\mathrm{d} \boldsymbol{C}_{0}(u)}{\mathrm{d} u}\right)\left(\frac{\mathrm{d} u}{\mathrm{~d} t}\right)
$$

Since the curve speed

$$
V\left(u_{k}\right)=\left\|\frac{\mathrm{d} C_{0}(u)}{\mathrm{d} t}\right\|_{u=u_{k}}
$$

The first derivative of $u$ with $t$ is obtained as

$$
\left.\frac{\mathrm{d} u}{\mathrm{~d} t}\right|_{t=t_{k}}=\frac{V\left(u_{k}\right)}{\left\|\frac{\mathrm{d} \boldsymbol{C}_{0}(u)}{\mathrm{d} u}\right\|_{u=u_{k}}}
$$

Therefore, the first-order interpolation algorithm is obtained by substituting Eq.(6) into Eq.(5), Eq. (5) can be processed as follows:

$$
u_{k+1}=u_{k}+\frac{T_{\mathrm{s}} V\left(u_{k}\right)}{\left\|\frac{\mathrm{d} \boldsymbol{C}_{0}(u)}{\mathrm{d} u}\right\|_{u=u_{k}}}
$$

The first and second derivative of $\boldsymbol{C}_{0}(u)$ with $u$ is obtained as

$$
\frac{\mathrm{d} \boldsymbol{C}_{0}(u)}{\mathrm{d} u}=\frac{\sum_{i=0}^{n} N_{i, p}^{\prime}(u) W_{i} \boldsymbol{P}_{i}}{\sum_{i=0}^{n} N_{i, p}(u) W_{i}}-\frac{\sum_{i=0}^{n} N_{i, p}^{\prime}(u) W_{i} \sum_{i=0}^{n} N_{i, p}(u) W_{i} \boldsymbol{P}_{i}}{\left(\sum_{i=0}^{n} N_{i, p}(u) W_{i}\right)^{2}}
$$

where the general algorithm for 1 st order derivative of $N_{i, p}(u)$ is

$$
N_{i, p}^{\prime}(u)=p\left[\frac{N_{i, p-1}(u)}{u_{i+p-1}-u_{i}}-\frac{N_{i+1, p-1}(u)}{u_{i+p}-u_{i+1}}\right]
$$

$\boldsymbol{C}_{0}\left(u_{k+1}\right)$ and $\boldsymbol{C}_{1}\left(u_{k+1}\right)$ are obtained by substituting the calculated $u_{k+1}$ into $\boldsymbol{C}_{0}(u)$ and $\boldsymbol{C}_{1}(u)$. Suppose the tool-axis unit vector is $\boldsymbol{T}_{k+1}$, so

$$
\boldsymbol{T}_{k+1}=\frac{C_{1}\left(u_{k+1}\right)-C_{0}\left(u_{k+1}\right)}{\left\|C_{1}\left(u_{k+1}\right)-C_{0}\left(u_{k+1}\right)\right\|}
$$

\section{Federate Controlling Method}

In this paper, it assumed that the federate controlling law is linear variable with acceleration $a$ and deceleration $-a$ and the destination controlling federate is $v$ as shown in Figure 2.

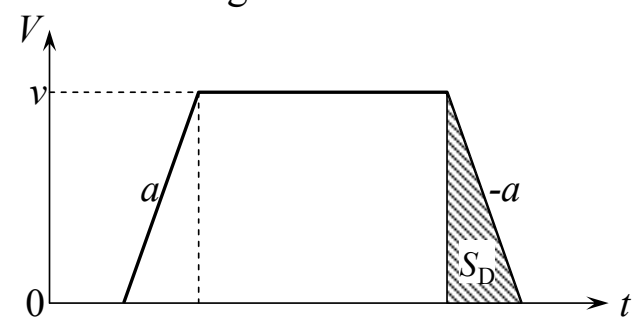

Figure 2. Model of linear feedrate controlling

For the acceleration process, the following method can be used

$$
V\left(u_{k}\right)=a t_{k}
$$

For the acceleration process, the key problem to be solved is evaluation of deceleration point. In Figure 2, $S_{\mathrm{D}}$, distance of deceleration, can be calculated as follows

$$
S_{\mathrm{D}}=\frac{v^{2}}{2 a}
$$


The distance of deceleration, which is also the length between deceleration point and destination point along the spline curve, can be calculated as follows

$$
\int_{u_{\mathrm{D}}}^{1}\left\|\frac{d \boldsymbol{C}_{0}(u)}{d u}\right\| d u=S_{\mathrm{D}}
$$

where $u_{\mathrm{D}}$ is corresponding parameter of the deceleration point. The following Newton-Rapson method is used to calculate $u_{\mathrm{D}}$.

If

$$
f(u)=\int_{u}^{1}\left\|\frac{d \boldsymbol{C}_{0}(u)}{d u}\right\| d u-S_{\mathrm{D}}
$$

we have

$$
f\left(u_{\mathrm{D}}\right)=0
$$

Assumed the initial value of $u_{\mathrm{D}}$ is 1 , that is, $f\left(u_{0}\right)=-S_{\mathrm{D}}$, then we have the following equation

$$
u_{i+1}=u_{i}-f\left(u_{i}\right) / f^{\prime}\left(u_{i}\right) \quad i \geq 0
$$

where $i$ is integer. Eq.(14) can be equivalent to the following formula

$$
u_{i+1}=u_{i}+\frac{f\left(u_{i}\right)}{\left\|\frac{d \boldsymbol{C}_{0}\left(u_{i}\right)}{d u}\right\|} \quad i \geq 0
$$

$f\left(u_{i}\right)$ in Eqs.(14) and (15) can be obtained using Simpson integral method.

\section{Realization}

The proposed 5-axis NURBS interpolation method has been realized in the developing 5-axis CNC system. The type of the 5-axis machine is table tilting/rotating. Fig. 4 shows that the CNC system is controlling cutting tool along a NURBS curve path to machine an impeller.

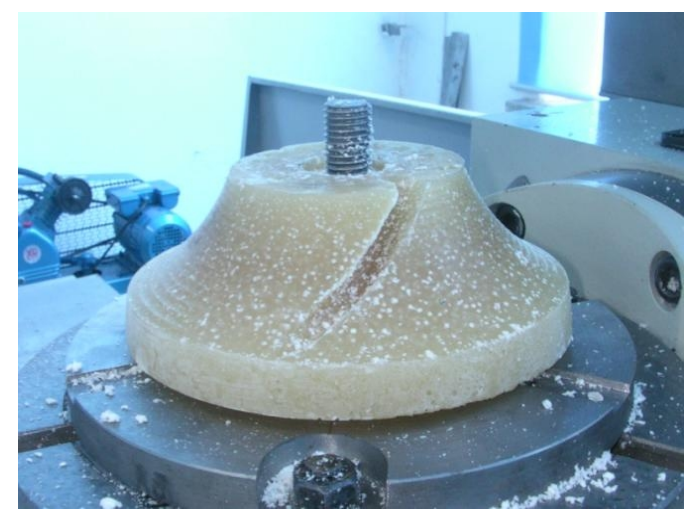

Figure 3. Application in itegral impeller machining

\section{Summary}

Thoroughly analyzing the defects of the existing 5-axis linear interpolation method used in the machining of sculptured surface, 5-axis NURBS interpolation method with location and federate controlling is presented and realized in a real-time CNC system. Different from the traditional machining method of the 5-axis linear interpolation, the proposed method can directly obtain a curve on a free-form surface. Consequently, the number of the NC code for the same machining path can be greatly decreased and machining efficiency and accuracy is also improved. 


\section{Acknowledgment}

This research is wholly supported by the project of National Natural Science Foundation of China numbered with 51275485 and the program for Science \& Technology Innovation Talents in Universities of Henan Province numbered with 13HASTIT036 through Zhengzhou Institute of Aeronautical Industry Management.

\section{References}

[1] L. Piegl, On NURBS: a survey, IEEE Computer Graphics \& Application, 11(1991) 55-71.

[2] M. Tikhon, et al, NURBS interpolator for constant material removal rate in open NC machine tools, International Journal of Machine Tools \& Manufacture, 44(2004) 237-245.

[3] B. Bahr, X. Xiao, and K. Krishnan, A real-time scheme of cubic parametric curve interpolations for CNC systems, Computers in Industry, 45(2001) 309-317.

[4] M. Cheng, M. Tsai, and J. Kuo, Real-time NURBS command generators for CNC servo controllers, International Journal of Machine Tools \& Manufacture, 42(2002) 801-813.

[5] R. E. Sorace, V. S. Reinhardt, and S. A. Vaughn, High-speed digital-to-RF converter, U.S. Patent 5668 842(1997).

[6] X. Zhiming, C. Jincheng, and F. Zhengjin, Performance evaluation of a real-time interpolation algorithm for NURBS curves, International Journal of Advanced Manufacturing Technology, 20(2002) 270-276.

[7] G. Qiyi, R. Zhang, and B. Greenway, Development and implementation of a NURBS curve motion interpolator, Robotics and Computer-Integrated Manufacturing, 14(1998) 27-36.

[8] M. Tsai, C. Cheng, and M. Cheng, A real-time NURBS surface interpolator for precision three-axis CNC machining, International Journal of Machine Tools \& Manufacture, 43(2003) 1217-1227. 